Cinémas

Revue d'études cinématographiques

Journal of Film Studies

\title{
La résistance du corps dans l'image cinématographique. La mort, le mythe et la sexualité dans le cinéma de Pasolini
}

\section{Silvestra Mariniello}

Volume 7, numéro 1-2, automne 1996

La Représentation du corps au cinéma

URI : https://id.erudit.org/iderudit/1000934ar

DOI : https://doi.org/10.7202/1000934ar

Aller au sommaire du numéro

Éditeur(s)

Cinémas

ISSN

1181-6945 (imprimé)

1705-6500 (numérique)

Découvrir la revue

Citer cet article

Mariniello, S. (1996). La résistance du corps dans l'image cinématographique. La mort, le mythe et la sexualité dans le cinéma de Pasolini. Cinémas, 7(1-2),

89-107. https://doi.org/10.7202/1000934ar
Résumé de l'article

L'irruption du « Tiers Monde » dans l'histoire occidentale, pendant les années soixante avec les luttes pour les indépendances africaines et la tiers-mondialisation des banlieues urbaines dans les pays industrialisés, révèlent l'obscénité du conformisme et la violence de la démoctatie. Le cinéma, qui offre une façon différente (toujours à réinventer, parce que toujours institutionnalisée) d'être-dans-le-monde, grâce à sa matérialité — faite de rythmes et de silences et de corps et de lieux - est le point de rupture possible : la brèche dans l'« entropie bourgeoise ". Cet article étudie quelques films de Pasolini qui « théorisent » la fonction révolutionnaire de la matérialité au cinéma. 


\title{
La résistance du corps dans l'image cinématographique. La mort, le mythe et la sexualité dans le cinéma de Pasolini
}

\section{Silvestra Mariniello}

\begin{abstract}
RÉSUMÉ
Lirruption du "Tiers Monde" dans l'histoire occidentale, pendant les années soixante avec les luttes pour les indépendances africaines et la tiers-mondialisation des banlieues urbaines dans les pays industrialisés, révèlent l'obscénité du conformisme et la violence de la démocratie. Le cinéma, qui offre une façon différente (toujours à réinventer, parce que toujours institutionnalisée) d'être-dans-le-monde, grâce à sa matérialité faite de rythmes et de silences et de corps et de lieux est le point de rupture possible: la brèche dans l'«entropie bourgeoise». Cet article étudie quelques films de Pasolini qui "théorisent " la fonction révolutionnaire de la matérialité au cinéma.
\end{abstract}

\section{ABSTRACT}

The breakthrough of the "Third World" in Western History, linked to African struggles for independance in the 1960s and to increasing "third-world" pockets in the cities of industrialized countries, reveals the obscenity of conformism and the violence of democracy. Because of its specific materiality - made of rythms and silences, bodies and places - film projects a different way (always to be reinvented because always institutionalised) of being-in-the-world and thus the possible point of breakdown: the leak in "bourgeois entropy." In this context, this article examines some of 
Pasolini's movies that "theorize" the revolutionary function of materiality in film.

Le corps, au moment où il devient matériau cinématographique - matériau pour la caméra et le montage — se fait théâtre de la médiation que la technique audiovisuelle opère en créant de nouvelles voies d'accès au "réel". La durée cinématographique, et en général la valeur du temps au cinéma, passent par le corps: le visage, les gestes, la figure, l'immobilité, la voix, le silence... Le rythme se produit en s'inscrivant dans le corps ou en l'effaçant.

Pasolini a très bien compris cette dimension du corps qu'il a "théorisée" dans ses notes avec la caméra, notamment les Notes pour une Orestie africaine, les Notes pour un film sur l'Inde, et les Sopraluoghi in Palestina et dans d'autres films comme La Rabbia, les films "mythologiques": Médée et Edipe Roi, la Trilogie de la vie et Salò. Par le corps et le rythme que sa matérialité impose, quand elle n'est pas refoulée dans la narration, d'autres formes de connaissance deviennent possibles.

Le corps, au cinéma, est le lieu privilégié de la résistance à la représentation et à la vision du monde qui en découle. Autrement dit, le corps devient le lieu de l'avènement d'une forme de connaissance qui se situe en dehors de la représentation et de ce que Pasolini, dans son poème "Il PCI ai giovani", avait défini comme l' " entropie bourgeoise ' ".

Mon article focalise l'intersection entre le corps et le rythme du film, à partir de quelques films de Pier Paolo Pasolini. Le rythme du film, qui se définit entre le temps et la matière, s'inscrit dans les corps - qu'il s'agisse du corps humain, animal, végétal ou architectural —, et se laisse modifier par leur résistance, par leur durée, jusqu'à ce que la narration, forme dominante de la représentation, s'ébranle et laisse la place à un discours non narratif et à d'autres modes de connaissance.

\section{Repérages}

Notes pour un film sur l'Inde est la recherche de matériaux lieux, visages, rythmes - pour un film sur l'Inde et la vérification de la "faisabilité" de ce film; il s'inspire de la légende d'un maharadjah se sacrifiant pour sauver de jeunes tigres affamés. 


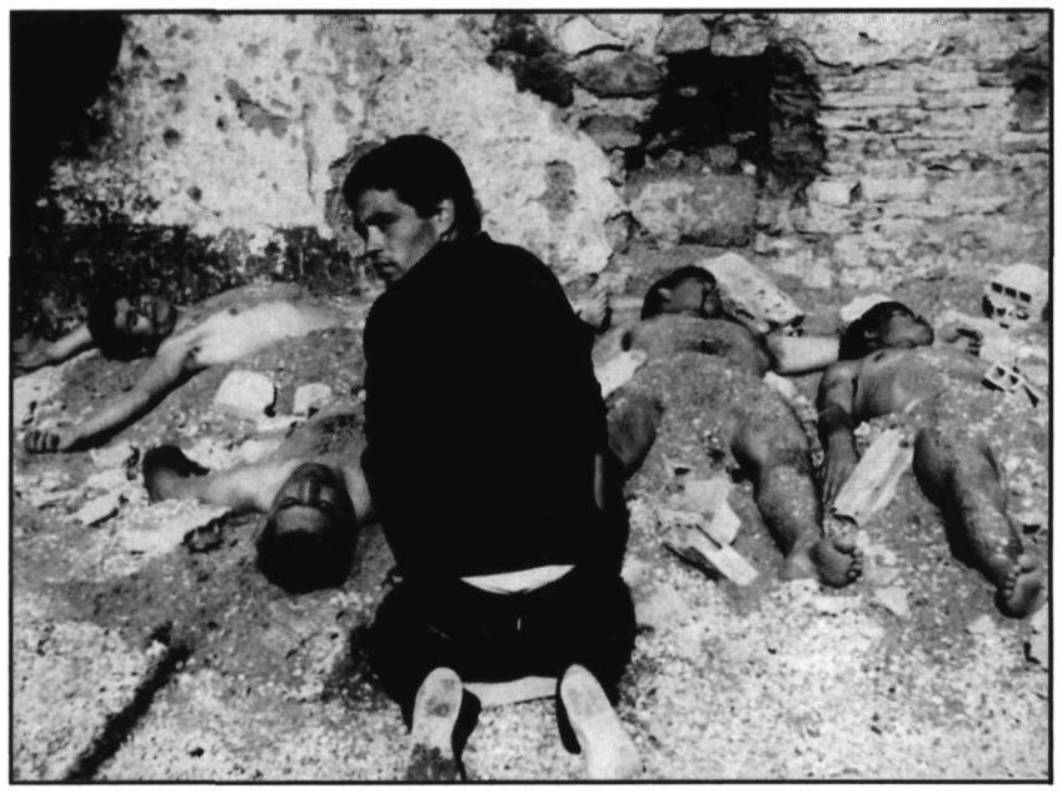

\section{Accatone de Pier Paolo Pasolini}

Collection Cinémathèque québécoise

Notes pour une Orestie africaine "raconte", de la même façon, la recherche de visages, de lieux, de gestes et d'objets pour un film inspiré de l'Orestie d'Eschyle, à situer en Afrique.

Sur le "Tiers Monde", Pasolini ne pouvait pas réaliser un film, mais seulement prendre des notes. Que veut dire «notes avec la caméra " et que signifie réaliser un film comme un "film à réaliser " ? Quelle relation se produit entre le "Tiers Monde " et cette "écriture" ouverte?

Selon Pasolini, le cinéma ne peut pas représenter l'Afrique, l'Inde, les "ghettos" nord-américains, mais le cinéma est une façon (la façon ) d'être-avec l'Afrique, l'Inde, le Sud, pas toujours géographique, des différentes situations mondiales; le cinéma est une façon de connaître, d'entrer en contact avec le "Tiers Monde ", et d'analyser sa relation avec lui. La technique audiovisuelle opère comme un moment actif de médiation entre l'Occident (on dirait plutôt aujourd'hui le Nord) et le "Tiers Monde" (le Sud), sans qu'elle se laisse réduire à un instrument de la pensće occidentale pour représenter la soi-disant "altérité". 
Quand Pasolini décide d' "écrire" avec la caméra son poème du "Tiers Monde", il ne l'aborde pas à partir de l'alternative entre un documentaire et un film de fiction. La seule possibilité, ce sont les notes. Le "Tiers Monde" semble opposer une résistance à la représentation: c'est la technique audiovisuelle ellemême qui rend possible une telle résistance; en d'autres mots, la résistance à la représentation est un produit de l'événementcinéma. Le médium cinématographique n'est pas un instrument pour représenter des idées ou des événements, mais il est l'avènement de ces événements et de ces idées.

D'une façon très semblable à celle de Pasolini, Jean Rouch réinvente le cinéma ethnologique. Dans un essai fondamental sur le cinéaste-ethnologue, intitulé "Jean Rouch ou le cinéma de la cruauté ", Réda Bensmaïa décrit une manière de faire du cinéma dans/sur le "Tiers Monde" qui pose les mêmes questions et les mêmes défis que les Notes pasoliniennes:

En d'autres termes, le cinéma comme technique intéresse moins Rouch comme instrument passif d'enregistrement du fait "brut" ou de la capitalisation d'un voir et d'un savoir à venir, que comme relais ou médium qui permet d'entrer en contact direct avec une réalité (culturelle, par exemple) qui ne présente au premier abord aucune homogénéité de structure avec la sienne (p. 51).

Les Notes de Pasolini, de Sopraluoghi in Palestina à l'Orestie africaine, se présentent comme une recherche en train de se faire, consacrée à établir ce "contact direct" dont Bensmaïa parle à propos de Rouch, avec une réalité différente de la réalité occidentale. La technique audiovisuelle a le pouvoir de bouleverser la connaissance, en la libérant de la domination de la pensée rationaliste bourgeoise (de droite et de gauche) qui a besoin de tout assimiler à ses propres modèles et valeurs. Il peut $y$ avoir de la connaissance sans "transparence"; le racisme inhérent au geste de "comprendre l'autre" en le jugeant d'après nos propres valeurs et donc en l'assimilant à notre propre culture est dénoncé de façon indirecte, par les Notes pasoliniennes.

Les cadres de l'Orestie sont bondés d'Africains. Ils sont bondés, pleins de gens; ils ne montrent pas, ne représentent pas des gens. Le cadrage, le rythme du film sont tels que le matériau - 
une personne, un mouvement, un objet, une rue - est plus important que son organisation dans une structure narrative. La nature des notes pour un film à réaliser permet au matériau brut de dominer l'écran. La voix off raconte l'histoire: "Vous tous connaissez l'histoire de l'Orestie d'Eschyle... " La caméra explore les visages, les corps, les sourires, les gestes. Quelqu'un cherche à échapper à l'objectif qui le suit, qui ne le laisse pas aller, qui le poursuit, qui l'"attrape». Les gens arrivent dans l'objectif et dans le cadre, bougent et réagissent à la caméra, regardent l'objectif, ne deviennent pas objet du regard ou du discours de quelqu'un. Bref, dans les Notes, le matériau cinématographique, qu'il s'agisse de l'Inde, de la Palestine, de l'Afrique ou de l'Orestie, ne se laisse pas réduire à un objet de représentation.

Pasolini cherche ses personnages: Agamemnons, Orestes, Clytemnestres, Cassandres, Pylades, maharadjahs, apôtres... Il ne s'agit pas de faire des bouts d'essai, il s'agit plutôt de la recherche d'un visage, d'un corps, d'une façon de marcher, mais aussi de ce que la caméra révèle de cette réalité physique. Généralement, pendant la réalisation d'un bout d'essai un acteur est observé, examiné par rapport au rôle qu'il doit jouer, par rapport à son adéquation au rôle. Le rôle est déjà défini, l'acteur doit lui correspondre. Considérons brièvement la recherche d'Agamemnon faite par Pasolini. On voit le gros plan d'un homme, on voit ensuite la même personne d'un point de vue à peine différent, il regarde dans une autre direction; finalement, on le voit en plan moyen : il est assis sur une chaise, au bord de la rue, il a une grande dignité, des gens restent debout près de lui. Il porte une tunique blanche nouée sur l'épaule, il regarde la caméra, "celui-ci pourrait être Agamemnon", dit la voix off "et celui-ci aussi ": la tête d'un vieux à moitié caché par des plantes, occupe la partie inférieure du cadre; en arrière-plan, quelqu'un travaille dans les champs; le cadre suivant nous montre un gros plan du vieillard. "Une autre variation, un autre possible Agamemnon ", dit encore la voix off. L'objectif s'approche au "zoom" d'un autre homme, grand, enveloppé dans une tunique blanche, debout sur le bord de la rue, "plus légendaire, plus mythique, cet homme est un Masai...". Un gros plan de l'homme qui porte plusieurs boucles d'oreille en forme de larges anneaux

La résistance du corps dans l'image cinématographique. La mort, le mythe et... 
prend tout l'écran. L'apparition matérielle des Agamemnons possibles, leurs histoires inscrites dans leurs regards, dans leur corps et dans les décors, pluralise le rôle Agamemnon et analyse le processus de représentation, les choix et les exclusions que celui-ci implique. La même chose arrive dans les autres notes, avec la recherche des possibles maharadjahs et des membres de la famille royale ou avec celle des lieux et des visages de la Palestine.

\section{La durée de la mort}

Le rythme est un des éléments les plus nouveaux et les plus révolutionnaires de ces Notes. Les différents segments du film à réaliser deviennent autonomes dans la durée: dans la lenteur et dans la répétition, le temps se concrétise et la matière envahit l'image, sortant des mailles de la structure narrative et de sa fonction "normalisante".

Un des moments les plus éloquents illustrant la façon dont le rythme travaille dans ces films est, peut-être, la séquence de la crémation à la fin des Notes pour un film sur l'Inde. En plan d'ensemble, une procession de gens qui chantent, vus parderrière, porte sur une civière un cadavre enveloppé dans des toiles. Cette image est suivie par une autre: une prise de vue des mêmes gens, cette fois pour en montrer les mains et les objets qu'elles portent, généralement des morceaux de bois; derrière la petite procession, il y a un camion, chargé de bois, avec une publicité pour Coca-Cola sur le panneau postérieur.

Maintenant les gens sont disposés en cercle autour du cadavre emmitouflé et déposé par terre. En plan rapproché, on voit le corps, dans l'herbe, et alternativement, les détails de la préparation du bûcher et du rituel qui l'accompagne. Les gestes sont plus importants que les visages. La voix off de Pasolini dit: "Un Occidental qui va en Inde a tout, mais en réalité il ne donne rien. L'Inde, au contraire, qui n'a rien, en réalité donne tout. Mais quoi?" (1991, p. $\left.168^{2}\right)$. Ce sont les derniers mots du film. En détail, on voit des fleurs et des feuilles éparpillées par terre. Le cadavre est déposé sur le bûcher, sur les fagots, encore enveloppé dans les toiles. En gros plan, plusieurs mains commencent à dérouler les toiles qui enveloppent le corps, la tête du cadavre 
bouge, poussée de part et d'autre par le mouvement. Par terre, un drap ouvert avec des rameaux. Le visage de la morte est découvert, des mains commencent à mettre du baume, une sorte de mousse blanche; la tête bouge encore, poussée à droite et à gauche par les mains qui couvrent le visage. Les jambes dénuées sont frottées avec la même mousse et sont ensuite recouvertes par les toiles. Les jambes sont attachées aux chevilles avec deux anneaux. Le gros plan d'un vieillard interrompt pour un moment la scène. D'autres rameaux sont déposés sur le cadavre. Quelqu'un s'essuie les mains. Un autre arrive pour allumer les fagots. La fumée commence à monter. La caméra parcourt en panoramique le groupe de gens réuni autour du bûcher. D'autres plans de la fumée et du feu se succèdent.

La matérialité de la mort envahit l'écran. Cette tête qui, sous la pression des mains laborieuses, bouge d'un mouvement qui n'est pas le sien, ce corps-chose entre les mains des vivants devient visible, presque palpable dans la durée de la séquence et dans le silence. La répétition des gestes n'a pas tellement la fonction de signifier une pratique, de raconter une histoire, de documenter sur un rituel. Il y a cela aussi, mais c'est surtout la matérialité de la mort dans la durée qui nous force à percevoir la fin du mouvement et la réification du corps. En même temps, le savoir des gestes, la continuité avec la tradition qu'ils expriment, leur appartenance à la culture et à l'expérience d'une communauté (même touchant désormais à sa fin, comme le panneau pour Coca-Cola sur le camion nous le suggère) transforment la mort "en des actes désespérés, mais tolérables de la vie" (Pasolini, 1990, p. $83^{3}$ ).

\section{La matière contre la normalité de la raison}

Pour comprendre et dénoncer la guerre qui appartient à l'ordre néocapitaliste et, en particulier, à la société des massesmédias dans laquelle on consomme quotidiennement l'illusion de la paix et de la normalité, Pasolini écrit un poème audiovisuel: La Rabbia. Il s'agit d'un film de montage composé d'images "documentaires" qui vont s'inscrire dans le texte en prose et en vers, qui les accompagne et qui les scande. Dans le mixage, la poésie composée sur les images semble les produire à 
nouveau, hors de leur caractère "documentaire " et du contexte de la chronique.

La banalité des images - même les plus tragiques deviennent banales dans le rythme des nouvelles organisées par secteurs d'information - est dépassée et dénaturée par les mots dans lesquels elles s'inscrivent. L'ordre chronologique ou logique, qui d'habitude lie les images dans un documentaire ou dans les nouvelles, est bouleversé. Il est remplacé par l'ordre "politico-poétique" d'un discours nouveau qui ne cherche pas à représenter la réalité, mais à s'inscrire dans sa matérialité, à la faire émerger dans sa complexité, à la produire sur l'écran.

Le rythme des images avec celui des vers en contrepoint et la nature fortement visuelle de ces derniers produisent une réalité historique qui met au défi l'organisation de la connaissance même dans sa qualité d'information, et la position du sujet dans le monde. Considérons, par exemple, les vers sur la couleur, "nouveau problème au monde". La couleur se matérialise et se visualise dans les termes spatiaux qui se répètent avec insistance dans le texte poétique - "elle s'appelle couleur la nouvelle extension du monde", "il faut accepter des étendues infinies de vies réelles", "agrandir la terre" — et dans l'évocation d'une différence vive, qui ne se laisse pas objectiver, faite de regards, d'amours, de danses. Une différence qui ne devient pas objet de connaissance, de représentation, mais qui provoque de nouvelles formes d'action en contaminant notre façon d'être-dans-lemonde. De nouveaux regards, de nouvelles façons d'aimer, de nouvelles danses nous deviennent familiers et les danses, les regards, l'amour sont eux-mêmes des modes de connaissance.

\section{L'écriture du mythe dans la durée et le silence}

Le silence joue un rôle important dans l'écriture du mythe, en créant un rythme étranger à la logique narrative de l'" entropie bourgeoise". Considérons, par exemple, la longue séquence du sacrifice humain et du rituel cannibale dans Médée.

L'effet le plus évident et le plus extraordinaire généré par cette longue séquence est celui de produire une "réalité" - en termes de sentiment du temps, conception de la vie humaine, rapport homme-nature - qui ne se rattache pas à la nôtre. Le 
silence, les sons, le rythme, les répétitions, la lumière, le paysage écrivent une pratique non logique, non verbalisable, qui ne se laisse pas juger selon les valeurs de la morale bourgeoise. En d'autres mots, la technique audiovisuelle libère la "préhistoire" de l'hégémonie de la vision bourgeoise du monde, et nous rend le mythe dans son "opacité", en tant que réalité dont la culture humaniste ne peut s'emparer, réalité qui ne se laisse pas "comprendre" (Glissant) et répartir en une dimension spirituelle et corporelle/matérielle. La tendance du spectateur à juger en ramenant tout à ses propres valeurs est frustrée, la désorientation est inévitable.

Dans toute la séquence, qui dure au moins 15 minutes, les personnes sont une partie du paysage, avec les montagnes, les animaux, les buissons, le ciel. Il n'y a pas de psychologie, même pas quand la caméra s'arrête sur Apsirte (le frère de Médée), pensif, souriant ou troublé... mystérieux et muet comme les blés, comme les pierres et les arbres rares, ou quand la caméra filme la jeune victime qui sourit, inconsciente, et qui après s'assombrit à cause d'un pressentiment, comme un animal sur le point d'être abattu.

Le rythme de la séquence est lent et la lenteur est faite de répétitions: d'un mouvement qui se produit par la somme de plans, par la somme de gestes, de regards et de couleurs. Les gens et les animaux se rendent sur le lieu du sacrifice. Le silence - seulement une musique à peine perceptible, métallique, semblable à celle des clochettes des animaux entendues au loin -, la lumière et l'échelle des plans inscrivent les gens et leurs attentes dans un paysage désertique, lunaire. Chaque geste se déroule en "temps réel ": dans ce cas, le temps du sacrifice et de la collectivité, non pas le temps dramatique et subjectif de la victime, par exemple, ou des bourreaux. À la musique se substitue ou s'ajoute le chant des cigales, obsessif, égal, répété. Mais considérons brièvement le moment culminant: celui de l'exécution de la victime et de l'accomplissement du rituel.

Le rythme de la séquence change soudainement grâce au mouvement rapide de la caméra qui suit les deux hommes se dirigeant, d'un pas expédié, chercher la victime préparée pour le sacrifice. En plan d'ensemble le jeune, vu de profil au début et 
ensuite de dos, se débat entre les deux hommes qui le conduisent à l'échafaud, il crie (ce sont les premiers sons humains de la séquence), mais on l'entend de loin. Les événements se précipitent grâce au mouvement de la caméra et au mouvement des silhouettes dans le cadre, ils conservent la nécessité tragique des calamités naturelles, ils ne se psychologisent pas dans un jeu de gros plans ni dans des dialogues. Le jeune est attaché aux poteaux, les bras ouverts, comme le Christ, la musique est un chant funèbre japonais qui ne sonne pas familier à l'oreille du spectateur occidental, mélangée au chant obsédant des cigales. Le ciel se teint de rose. Le jeune ne se révolte plus, il n'émet plus aucun son; les gros plans d'Apsirte et de Médée s'alternent, puis suit la mise à mort, par étouffement, lente, inéluctable, silencieuse. Elle n'est pas suivie par les clameurs de la foule "sauvage", par la libération d'énergies "orgiaques", vues par l'œil "civilisé" de celui qui raconte le mythe primitif. Quelqu'un, avec un casque surmonté par la tête d'un animal, s'approche, muni d'une hache, il libère le corps des cordes qui le tenaient lié et commence à le couper en morceaux. Les gens s'approchent, leurs visages sont paisibles comme ceux des moutons ou des ânes des premiers cadres de la séquence. Des mains équipées de bols, différents quant à la forme et à la grandeur, s'avancent pour recevoir le sang de la victime. Nous voyons les mains, mais pas les visages. Une procession de gens, bien rangés, s'éloigne rapidement, en plan d'ensemble, dans un paysage de rochers et de sable. La caméra quitte les gens et parcourt le paysage, les habitations-termitières et les plantes, "miracle sublime" dans le désert. En plongée, l'objectif cadre les gens qui se dispersent en plan d'ensemble, en direction de la verdure. Le gros plan d'un garçon, l'expression énigmatique, se dissout dans un panoramique des visages suivi par le détail des mains qui performent le rituel. On voit surtout les gestes, les actions qui accomplissent le rituel. Le sacrifice et le rituel restent à tout moment collectifs, ils ne s'individualisent pas dans la perversion ou dans la pitié, ni dans la démonisation de la foule qui, à son tour, ne s'individualise pas, ne devient pas sujet psychologique. 


\section{Le matérialisme de l'idéologie implicite}

La Trilogie aussi devient le moment de la recherche d'un nouveau langage qui, à travers le rythme imposé par la matière, rompt le schéma de la représentation. Pasolini parle d'idéologie "implicite" pour décrire la stratégie adoptée dans ces films: la politique du film est entièrement inscrite dans le matérialisme des images et des sons. La révolte contre l'idéologie dominante, en particulier contre la philosophie de la consommation, passe par les corps, les rythmes, les sons (y compris le dialecte et les chansons populaires), les lieux qui, à travers leur présence physique, renvoient de façon critique aux images et aux valeurs de la société néocapitaliste. "Un personnage du Decameron est exactement le contraire d'un personnage qu'on voit dans les programmes de la télévision ou dans les soi-disant films consolatoires", avait dit Pasolini dans l'un de ses entretiens avec la revue Filmcritica (cité par Magrelli, p. 105).

La dimension physique du personnage, du lieu, du geste devient particulièrement importante et le cinéma est exploré et compris surtout comme médium qui peut rendre et écrire une telle dimension physique. De là, l'urgence presque obsessionnelle de la descente sur les lieux, de la recherche infatigable des visages et des corps. Pasolini parle longuement de cela dans un texte important publié dans Écrits sur le cinéma, qui relate ses expériences de voyage en Eritrée et ensuite au Yémen, à la recherche du matériau humain, géographique, architectural et pictural qui devait rendre possible Les Mille et une nuits.

La sexualité, centrale dans les trois films de La Trilogie, s'inscrit dans le matérialisme pasolinien, dans cette valorisation de la présence physique qui, venant à habiter l'écran, nie l'«irréalité" " de l'expérience quotidienne dans les sociétés occidentales et modernisées. Dans Théorème, Porcherie et Salò, la sexualité devient langage et se soustrait au contrôle de la vision bourgeoise du monde en se chargeant d'enjeux politiques nouveaux. Ici, le projet pasolinien de prendre la sexualité comme le lieu critique d'où considérer le système social qui nous "emprisonne" assume des caractères différents. Dans La Trilogie, nous ne sommes pas en présence d'une sexualité codifiée, dans laquelle on puisse identifier, comme Barthes le faisait pour Sade et 
comme on peut en partie le faire pour Théorème et Salò, les éléments constitutifs du code et même définir une grammaire érotique. Le résultat est encore celui de dépouiller la sexualité du regard bourgeois qui la transforme soit en amour, soit en perversion et il est atteint encore par l'élimination de toute tension psychologique entre deux êtres, par l'élimination de la psychologie, pivot de la vision bourgeoise centrée sur l'individu-sujet. Mais les modalités de cette "écriture" de la sexualité sont différentes. La sexualité de La Trilogie est tout inscrite dans la dimension physique des personnages: bruyants, joyeux, replets ou minces, barbus, édentés, décoiffés, souriants ou râleurs, beaux d'une beauté non "régulière", abrutis par la misère ou le vin, timides ou effrontés et, surtout, tout à fait insouciants de leur aspect: scandaleuse provocation dans une société surtout préoccupée de son propre aspect. Dans Théorème, Porcherie et Salò, les personnages sont presque des "simulacres", des formes diaphanes et silencieuses, des rôles, des symboles ou encore des nombres interchangeables dans le jeu impitoyable du Pouvoir.

Le rythme de ces films constituant La Trilogie produit une dimension chorale qui nie, justement, les individus et les psychologies; qui fait des personnages les éléments d'un tout, qui privilégie le "peuple" sur l'individu, pour reprendre les mots prononcés par Pasolini à propos de la destruction de Sana'a ${ }^{4}$. En effet, le metteur en scène constatait, à propos de la petite ville yéménite, que la "démocratie formelle » importée par les Russes et les Américains "enseigne à respecter l'individu, mais elle n'enseigne pas à respecter la volonté d'un peuple " (Pasolini, 1991, p. 268).

La choralité de La Trilogie est produite par les plans d'ensemble; par la mise en scène d'un grand nombre de personnages, différenciés dans l'aspect et les métiers, et rapprochés par la ressemblance de leurs histoires; par leur inscription dans l'espace architectural qui n'est pas un simple décor, mais plutôt une sorte de protagoniste de l'histoire constitué par la place du marché, la cathédrale, le cloître, l'auberge, les ruelles; par l'omniprésence des bruits: chansons populaires, cris des vendeurs au marché, chuchotements, soupirs, rots, pets - même les dialogues deviennent des "bruits" en s'inscrivant dans cette choralité; par le montage accéléré qui n’a presque jamais recours 
au fondu, exception faite pour La Fleur des mille et une nuits et même ici, pas tellement pour marquer le passage d'une histoire à l'autre, mais pour marquer les évolutions à l'intérieur de la même histoire. Les épisodes se suivent dans la continuité d'un tout, sans ponctuation, comme dans la vie.

\section{La sexualité comme langage}

Salò est la mise en scène de la perpétration de la part du Pouvoir du génocide culturel et physique d'un monde, et la mise en scène de la perpétuation de l'infamie et de la violence jusqu'à ce que celle-ci devienne mécanique; en d'autres mots, il s'agit de la mise en scène d'une véritable technologie de la violence. Salò est aussi l'écriture de la révolte possible contre le Pouvoir et contre la culture qu'il engendre.

Pourquoi Sade? Au-delà du défi au conformisme intellectuel de droite et de gauche, prêt à passer sous silence l'infamie du Pouvoir, le dialogue avec Sade permet à Pasolini de développer une intuition qui est déjà à la base d'une grande partie de son travail — de Théorème à Porcherie, à La Trilogie (même Petrole participe de la même recherche) —, l'intuition de prendre la sexualité comme lieu critique d'où considérer la construction sociale, à laquelle nous participons, et ses limites.

En parlant de Théorème, Pasolini avait dit:

Dans le monde d'aujourd'hui, l'Individu, en proie à l'aliénation, vit avec une fausse idée de soi, de façon non authentique. Le rapport entre authenticité et inauthenticité est impossible sur le plan de la communication linguistique: en effet, le jeune invité ne parle pas avec les autres personnages, il ne cherche pas à les convaincre avec des mots, mais il a avec eux tous un rapport d'amour (cité par Naldini, p. 3265).

La langue est l'ossature même de l'"entropie bourgeoise". La révolution doit passer par d'autres médias. La sexualité, interprétée dans ces films comme un langage, devient l'issue de la vision bourgeoise du monde. En d'autres mots, il est possible de libérer l'érotisme des superpositions idéologiques qui l'ont transformé chaque fois en "amour" ou en "perversion", de le ramener à la matérialité des corps, des gestes, des silences et d'en faire le lieu 
d'implosion de la vision bourgeoise du monde. Le cinéma, grâce à sa nature audiovisuelle, semble être le médium idéal pour une telle opération.

Le sexe, dans Salò, n'est pas "la métaphore du pouvoir fasciste" comme le dit Macciocchi dans son néanmoins important essai Quatre Hérésies Cardinales pour Pasolini. Le fait de lire le sexe comme la métaphore du pouvoir fasciste oblige en effet à rester dans le même cadre idéologique qui produit un tel pouvoir $^{6}$. La métaphore sexuelle renvoit à un référent: le pouvoir fasciste, objet de réprobation de la part de la morale démocratique et progressiste. Cette dernière, de son côté, reste intacte, ou même est confirmée dans sa positivité devant tant d'horreur. En d'autres mots, la vision bourgeoise du monde, même si elle est progressiste, ne se met pas en question en tant que vision du monde basée sur la représentation. Le fascisme n'est pas le référent de l'allégorie sexuelle de Salò (comme on le verra plus particulièrement ensuite). La grammaire érotique que Pasolini reprend de Sade renvoie à la grammaire du pouvoir fasciste avec sa structure hiérarchique et ses modes d'articulation et tous les deux renvoient au pouvoir néocapitaliste qui réduit le corps à un accessoire insignifiant et jetable. Le renvoi entre les différents discours - celui du fasciste, du sadien et du néocapitaliste (propre à l'économie de la consommation) - est le moment où la conscience critique de l'histoire se produit. La dynamique entre ces discours est médiatisée par une sexualité qui se fait langage en se soustrayant au langage verbal.

Dans l'œuvre de Pasolini (et de Sade), l'érotisme se soustrait à la "parole perpétuellement allusive" (Barthes, p. 32) dont il fait l’objet dans l'expérience bourgeoise; il est proclamé, révélé dans ses pratiques, dépouillé du désir, des attentes, des suggestions, des psychologismes... Le défi et l'importance de cette procédure demeurent dans le fait que la pratique érotique et le sexe se séparent de la vision bourgeoise du monde et nous offrent l'opportunité vertigineuse d'un autre point de vue qui nous remet nécessairement en question.

Dans son Sade, Fourier, Loyola, Barthes pose, avec grande lucidité, la question du langage érotique et des définitions erronées qu'on en donne: "Sade est un auteur "érotique", on nous 
le dit sans cesse. Mais qu'est-ce que l'érotisme?» (p. 31). Chez Sade, il s'agit d'une langue "agie, non plus parlée ", dira Barthes, tandis que dans notre société il s'agit surtout d'un fait de parole: allusive, indirecte, mais indispensable à la production de ce que nous considérons être l'érotisme. Barthes décrit le code érotique de Sade, avec ses unités de première et de deuxième articulation, jusqu'à définir une véritable grammaire érotique. Plus loin dans son essai, en répliquant aux différentes critiques faites à Sade, Barthes dit quelque chose de très important: la société qui le met à l'index

[...] ne voit dans l'œuvre de Sade que l'appel du référent; pour elle, le mot n'est qu'une vitre qui donne sur le réel; le procès créatif qu'elle imagine et sur lequel elle fonde ses lois n'a que deux termes: le "réel" et son expression. La condamnation légale portée contre Sade est donc fondée sur un certain système de la littérature et ce système est celui du réalisme: il postule que la littérature "représente", "figure", "imite"; que c'est la conformité de cette imitation qui s'offre au jugement, esthétique si l'objet en est touchant, instructif ou pénal, s'il est monstrueux; qu'enfin imiter c'est persuader, entraîner: vue d'école, dans laquelle pourtant s'engage toute une société, avec ses institutions.

$[\ldots]$

La fonction du discours n'est pas en effet de "faire peur, honte, envie, impression, etc. ", mais de concevoir l'inconcevable, c'est-à-dire de ne rien laisser en dehors de la parole et de ne concéder au monde aucun ineffable: tel est, semble-t-il, le mot d'ordre qui se répète tout au long de la cité sadienne, de la Bastille, où Sade n'exista que par la parole, au château de Silling, sanctuaire, non de la débauche, mais de l'«histoire» (p. 4142).

Concevoir l'inconcevable, démanteler le système idéologique sur lequel toute une société avec ses institutions se fonde est aussi le travail de Pasolini. Le référent n'est pas l'élément important du film, l'attention se concentre sur les figures, sur la construction rhétorique, sur le renvoi entre les différents discours dans une tentative de réécrire l'histoire. Salò est, comme Théorème et

La résistance du corps dans l'image cinématographique. La mort, le mythe et... 
Porcherie, une allégorie tragique: une figure qui ne renvoie pas à un référent, mais à d'autres constructions discursives, à leur fonction et à leur différence.

"Pourquoi ces montagnes de cadavres des camps nazis que nous entrevoyons aujourd'hui parfois à la télévision " (Macciocchi, p. 131), ou les corps de Vietnamiens noyés en essayant de s'enfuir du Vietnam "libérée, ou les cadavres des Hutus et Tutsis massacrés ne nous sont-ils pas intolérables? "L'histoire apparaît comme un trafic ininterrompu de cadavres " (p. 132), mais nous acceptons de regarder cela chaque jour; de même, la pornographie a ses propres canaux de diffusion, et est intégrée aux autres pratiques sociales: "L'intolérable est donc que l'art fouille dans la perversion du pouvoir. Que l'artiste en donne sa propre interprétation: c'est cela qu'on juge "pornographique" " (p. 131). Mais je voudrais ajouter ceci : le vrai scandale et la vraie force du film demeurent dans le fait que cette dénonciation de la perversion du Pouvoir passe par un langage non contrôlable, qui met en question les bases de notre compréhension du monde, la possibilité même de l'interprétation. Dans cette opération radicale se développent la fonction pédagogique de Salò et la critique de Pasolini contre le "fanatisme idolâtre de l'État" (p. 132) partagé par la droite et la gauche sur lequel Antonietta Macciocchi insiste avec grande lucidité.

\section{Le silence, l'action, le sang ou la mort}

Le sens de toute la recherche pasolinienne est le suivant: comment habiter la fluidité de l'histoire, comment changer le cours de l'histoire de l'intérieur, en respectant ses contradictions, en n'essayant pas de tout ramener à la raison, en acceptant de ne pas pouvoir en éliminer l'incertitude.

Habiter l'histoire signifie aussi, dans le cas de Pasolini, travailler dans le cinéma, choisir l'audiovisuel pour connaître d'une autre façon le monde et ses temporalités, comprendre et inventer la valeur des silences, de la matérialité de l'image ("J'attache une grande importance aux visages et il est impossible de tricher avec eux", cité par Duflot, p. 121), travailler sur la notion d'exemple avec la conscience du devenir-exemple de l'action cinématographique. Comme dans la vie, nous nous construisons 
à l'exemple des personnes proches de nous, en répétant, même sans le savoir, leurs gestes, leurs discours, leurs actes quotidiens, au cinéma nous sommes exposés à un phénomène tout à fait semblable qui passe par la matérialité de l'image, par la compréhension, même inconsciente, de ses rythmes.

Je voudrais conclure avec la discussion de quelques passages du dialogue entre Julian et Spinoza dans la tragédie Porcherie, qui me semblent essentiels pour comprendre ce qui est en jeu dans cette recherche de Pasolini. La rencontre avec Spinoza, passage fondamental dans la tragédie homonyme de 1966, n'a pas été inclue dans le film; elle devait se passer vers la fin, quand Julian se rend pour la dernière fois à la porcherie.

À la méfiance de Julian, qui suppose chez le philosophe une volonté de le condamner, Spinoza répond:

Je devrai donc être ici pour te dire: "Libère-toi de l'esclavage des sentiments, Julian, au moyen de la raison: et retourne dès lors parmi les hommes, si tu veux être un homme!" Et j'ajouterais : "Va là-bas, à l'endroit où le cocktail de la fusion bat son plein : tu y trouveras, certes, le compromis, mais aussi la liberté de l'hérésie et de la révolution" (Pasolini, 1995, p. 376).

Tout appartient au même cadre: le pouvoir et la révolte contre le pouvoir. Le rationalisme est à la base des deux. Mais le rationalisme n'avait pas pu liquider Dieu.

SPINOZA. - Étrange contradiction chez ce Juif de 40 ans! Expliquer Dieu par la Raison : je veux dire, par la Raison qui était déjà scientifique - et bourgeoise (Pasolini, 1995, p. 376)!

Nous ne sommes pas en présence de l'éloge de l'irrationnel, du refus de la raison, mais de la critique de la Raison en tant que construction historique. $L a$ raison n'existe pas, il y a un concept de raison qui s'est produit dans la conjoncture historique de l'émergence de la bourgeoisie et qu'on a voulu dépouiller de cette dimension historique pour le faire devenir absolu, mais il y a d'autres manières de concevoir la raison.

La décision finale de Julian - se laisser dévorer par les cochons - est décrite par Spinoza comme une décision historique,

La résistance du corps dans l'image cinématographique. La mort, le mythe et... 
la décision d'une époque. Par la prise de conscience de l'historicité de la révolte, la nécessité s'impose de trouver d'autres formes de révolte.

SPINOZA. - Appelle comme tu veux ta façon de communiquer, que ton père désigne par la formule : "ni obéissance, ni désobéissance"; le fait est que beaucoup de saints, par exemple, ont prêché sans dire un seul mot - par le silence, l'action, le sang ou la mort. [...] C'est pour témoigner de ce type de langage qu'aucune Raison ne peut expliquer, fût-ce en se contredisant, que tu as été appelé.

JULIAN. - Je ne veux pas être réduit au rôle de cobaye, fût-ce de ton Éthique.

SPINOZA. - Julian, tu n'as donc pas compris? Je suis ici pour l'abjurer. Ce n'était qu'un livre - comme le Don Quichotte, comme la Monadologie, ou les Principia mathematica: livres sublimes, si l'on veut: et pourtant issus d'un monde qui devait donner naissance, en fin de compte, à ton père humaniste et à son associé technocrate. Bien plus, ces œuvres n'ont servi qu'à les glorifier eux; à avaliser leur histoire à eux. C'est vrai : la Raison (leur Raison) m’a servi à expliquer Dieu. Mais dès qu'elle a expliqué Dieu, la Raison a accompli son devoir et doit se nier: il ne doit rester que Dieu, rien d'autre que Dieu (Pasolini, 1995, p. 378-379).

Mais Dieu, ce "Dieu qui ne console pas" (p. 379) est l'histoire et le langage de l'exemple, comme celui des saints qui ont prêché avec le silence, avec l'action et le sang, et il est aussi le cinéma. Le cinéma comme réécriture de l'histoire, comme réinvention à partir de sa matérialité, justement, d'une façon d'être dans le monde.

\section{Université de Montréal}

\section{NOTES}

1 L'entropie est le retour irréversible de la bourgeoisie à elle-même.

2 Ma traduction.

3 Ma traduction. 
4 En 1970, Pasolini avait tourné un court métrage contenant un appel à I'UNESCO pour sauver Sana'a.

5 Ma traduction.

6 Il s'agit plutôt d'une allégorie que d'une métaphore, dont les conséquences politiques sont importantes, comme on le verra plus loin.

\section{OUVRAGES CITÉS}

Barthes, Roland. Sade, Fournier, Loyola, Paris: Seuil, 1971.

Bensmaïa, Réda. "Jean Rouch ou le cinéma de la cruauté ", CinémAction, n" 17 (1982), p. 50-58.

Duflot, J. Les dernières paroles d'un impie. Paris: Belfond, 1970.

Glissant, E. "Le chaos-monde, l'oral et l'écrit ", Écrire la "parole de nuit». Paris: Gallimard, 1994.

Macciocchi, Antonietta. "Quatre Hérésies cardinales pour Pasolini ”, Pasolini. Paris: Grasset, 1980.

Naldini, N. Pasolini una vita. Torino: Einaudi, 1989.

Pasolini, Pier Paolo. Écrits sur le cinéma. Lyon: Presses universitaires de Lyon, 1987.

Pasolini, Pier Paolo. L'Odore dell'India. Parma: Guanda, 1990.

Pasolini, Pier Paolo. Le Regole di unillusione. Roma: Fondo Pier Paolo Pasolini, 1991.

Pasolini, Pier Paolo. Théatre. Babel, 1995.

La résistance du corps dans l'image cinématographique. La mort, le mythe et... 107 